\title{
Ethical challenges in medical practice in the context of Coronavirus disease 2019 in Nepal
}

\author{
Dr Nuwadatta Subedi (i) \\ Associate Professor \\ Department of Forensic Medicine, Gandaki Medical College, Pokhara \\ E mail: drndsubedi@gmail.com \\ DOI: $10.3126 /$ jgmen.v|3il.29257
}

The Coronavirus disease 2019 (COVID-19) pandemic has posed unexpected challenges to government, health care providers, policy makers and people globally. This disease is new, highly contagious and has no definite treatment, vaccine or preexisting immunity. The governments of different nations globally are using several strategies to contain and treat the disease, but the situation has been a nightmare for all till date. There are deep impacts in global economy due to the lockdown and decreased productivity can leave its effect for years to come. ${ }^{1}$

Journal of GANDAKI MEDICAL COLLEGENEPAL (J-GMC-N)

J-GMC-N | Volume 12| Issue 02| July-December 2019
The pandemic has posed a greater challenge in health sector and has triggered many ethical issues as well. Medical ethics is a set of moral principles which guides the clinical practice in the management of patients. It also applies to scientific research in the field of health care. Medical ethics is based on a cluster of principles which are referred to in case of any ethical confusion during management of patients. The "Principles of Biomedical Ethics" proposed by Tom L. Beauchamp and James F. Childress in 1977 has been regarded as the most practical in the field of medical practice and research particularly in Anglophone scholarship. ${ }^{2}$ The four principles of health care ethics are autonomy, beneficence, non-maleficence, and justice. Though the principles have widely been accepted, some shortcomings have also been commented by authors, such as 'thick in status but thin in content' by Lee MJH. ${ }^{2}$

Health care providers deal with human suffering and there might be ethical dilemmas even under normal conditions, but they can be solved with minor workouts. There will be greater challenges when dealing with life threatening diseases and conditions, particularly when the patients are unable to make their own decisions. Whenever there is ethical dilemma during the management of a patient, healthcare providers can seek opinion from the ethical committee of the institutes whosoever can analyze the case in the particular context in team and provide best solution to the scenario. The service can also be extended to the family members or legal guardians in making informed choices to avoid harm to the patient and fair action. ${ }^{3}$

Though the ethical care is patient-centered, the conditions during the public health emergency like COVID 19 pandemic may disrupt or at least alter the usual process of ethically serving a patient. ${ }^{3}$ During the pandemics, protection of a larger population gets preference over individual rights and privileges and prudent use of resources need to be done. When the resources are limited, the patients who could be protected during the normal conditions might not get adequate treatment and care as the patient-centered medical care is diverted to public-centered during the public health emergencies. In such a crisis, Public health ethics guide health care providers to 
balance the challenging issues between the needs of the individual and of the public at large.

There have been concerns as the patients who have higher risk such as elderly and those having several underlying diseases and conditions are getting refused for treatment in favor for those whose prognosis is predicted to be better. This problem has been already experienced by health care providers in countries where the serious cases have been very high in number.

There have been recent developments in the health care facilities and availability of trained manpower in Nepal, but it is mainly restricted to major urban areas only. The unequal distribution of hospitals is an alarming situation in Nepal even for the regular provision of health care during normal conditions. In the current pandemic crisis, the situation can be grave if there is drastic surge of critical COVID 19 patients. There would be serious ethical and as well managerial challenge in our context.

The medical care is focused to protect greater population with strategic changes during the pandemics like COVID 19. But the rights of individuals who are in serious need of health care facilities due to other health reasons should not be neglected. Patients are afraid to go to hospitals in a fear of infection and opting traditional measures of treatment at home. It has mainly affected the pregnant mothers who fear to visit hospitals for regular antenatal care. The chances of infection could be more when medical or surgical procedures need to be done. The patients themselves might contract disease in the hospital from other patients and health care providers. The situation is important and should be dealt tactfully.

Health care providers are also vulnerable to distress due to increased workload and risk of contagious infection in the context of COVID 19 pandemic. The management of health institutions should be cautious to protect the frontline workers. The scarcity of proper Personnel Protective Equipment (PPE) poses risk of disease transmission to them. It is also challenging ethically in terms of putting one's own health into risk to care for others. Many health care providers have been infected with COVID 19 and even succumbed to the disease in different parts of the world.

Nepal Medical Council(NMC) has prepared interim guideline for infection prevention and control when COVID-19 is suspected. The document mentions the safety that the health care providers need during the management. It also includes organizational preparedness and the safety of visitors, attendants, care of instruments and laundry. The possible scarcity of PPEs, including the $\mathrm{N} 95$ face masks and the ways to reuse of the mask are also mentioned. The guideline needs to be followed by the health care professionals in order to keep themselves and other persons directly or indirectly involved in the management of the known or suspected COVID 19 patients safe. The guideline has been instrumental as an important document to follow in the present context. It needs to be updated with the addition of latest evidence of the nature of the disease. ${ }^{4}$

Nepal Medical Council has also published professional ethical guidelines in the management of patients in the context of COVID 19 in Nepal on $20^{\text {th }}$ March, 2020. The challenges may differ in different context based on the availability of resources and trained manpower. Therefore, the local guideline in the context of Nepal was essential. The guideline mentions the need of public health ethics in order to protect greater community at large. Along with, the ethical aspects and rights of individual patients 
in the context of current pandemic is included. The guidelines recommend avoiding elective medical and dental procedures during the pandemic as far as possible. This could breach the rights of individual patients but is needed to prevent the greater public getting infected. The document is also in favor of the lockdown conducted by the government of Nepal. The protection of health care professionals is emphasized and their responsibilities for protection of public is also highlighted. Although the document lacks in comprehensiveness in application of principles of public health ethics, the publication of guideline itself is praiseworthy in the current crisis. It recommends following the national and international guidelines in infection control as well to follow the established standard operating procedures in case basis. The health care providers should refer to the guideline to solve ethical dilemmas in the local context. ${ }^{5}$

The characteristics of COVID 19 is still getting explored and many facts are yet to come, but the current pandemic has already posed a great threat to the people globally. Everyone is responsible from their aspects to face the global crisis. Health care providers are highly responsible to heal the sufferers and to make critical decisions. The ethical aspects in management of patients should be followed properly to make rational decisions.

\section{REFERENCES}

1. Hurford JE. The BMA COVID-19 ethical guidance: a legal analysis. New Bioeth. 2020 May 2:1-14. DOI: 10.1080/20502877.2020.1762027

2. Lee MJH. The problem of "thick in status, thin in content" in Beauchamp and Childress' principlism. J Med Ethics. 2010;36(9):525-8. DOI: 10.1136/ jme.2009.031054 PMID: 20817819.

3. Berlinger N, Wynia M, Powell T, Micah Hester D, Milliken A, Fabi R, et al. Ethical Framework for Health Care Institutions Responding to Novel Coronavirus SARS-CoV-2 (COVID-19). Hast Cent. 2020;2:1-12.

4. Nepal Medical Council. Nepal Medical Council interim guidance for infection prevention and control when COVID-19 is suspected. April 5 2020. Available from: https://nmc.org.np/files/4/NMC\%20

COVID-19\%20Interim\%20Guideline\%20for\%20prevention.pdf [Accessed May 31 2020].

5. Nepal Medical Council. Professional ethical guidelines during COVID-19 pandemic. March 2020. Available from: http://simon. org.np/ uploads/downloads/file_dIOXrQo0bjaMKCikrfIN.pdf. [Accessed May 31 2020]. 